\title{
Research Paper: Waist Circumference, a Clinical Measurement for the Activity of Transversus Abdominis
}

\author{
Mojtaba Kamyab'* (D), Brendan McHugh², Roy Bowers² ${ }^{1}$ (D) \\ 1. Department of Orthotics and Prosthetics, California State University Dominguez Hills, USA. \\ 2. Department of Biomedical Engineering, National Centre for Prosthetics and Orthotics, University of Strathclyde, United Kingdom.
}

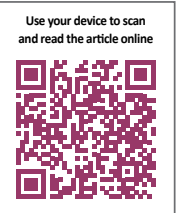

Citation: Kamyab M, McHugh B, Bowers R. Waist Circumference, a Clinical Measurement for the Activity of Transversus Abdominis. Iranian Rehabilitation Journal. 2021; 19(3):273-278. http://dx.doi.org/10.32598/irj.19.3.1391.1

http://dx.doi.org/10.32598/irj.19.3.1391.1

Article info:

Received: 06 Apr 2021

Accepted: 06 Aug 2021

Available Online: 01 Sep 2021

\section{Keywords:}

Waist circumference, Clinical measurement, Transversus abdominis

\section{ABSTRACT}

Objectives: Improving the timing and endurance of the deep abdominal muscles, notably transversus abdominis (TrA), is a known protocol for improving spinal stability. $\operatorname{Tr} A$ is the deepest abdominal muscle, and monitoring its activity is a difficult task. Ultrasound and pressure biofeedback have been employed for monitoring the activity of $\operatorname{Tr} A$; however, these methods are expensive, not always available in all clinical settings, and their application requires formal training. The purpose of this study was to examine the use of a broadly known method, i.e., changes in the waist circumference, to monitor the activity of TrA.

Methods: The study was carried out on 14 subjects following a pilot study of 5 participants. The thickness of TrA measured by ultrasound was considered the standard indicator for activity of TrA and was compared with simultaneous measurement of waist circumference.

Results: A significant criterion validity between the thickness of $\operatorname{Tr} A$ and the waist circumference was established (Pearson correlation $=-0.71, \mathrm{P}=0.001$ ), indicating an inverse relationship between changes in the thickness of the transversus abdominis muscle and waist circumference.

Discussion: This result confirmed the hypothesis that changes in the waist circumference could be employed as a suitable indicator for the activity of TrA.

\section{* Corresponding Author:}

Mojtaba Kamyab, PhD.

Address: Department of Orthotics and Prosthetics, California State University Dominguez Hills, USA.

Tel: +98 (912) 5068770

E-mail: mojtaba.kamyab@gmail.com 


\section{Highlights}

- Ultrasound and biofeedback have been standard method for monitoring the activity of $\operatorname{TrA}$.

- Waist circumference fluctuation is comparable with ultrasound in monitoring TrA thickness.

- Measuring changes of the waist circumference helps in monitoring the activity of TrA.

\section{Plain Language Summary}

One of key muscles in abdominal region is Transversus Abdominis (TrA). The activity of this muscle is important in trunk stability. Within the present study the authors investigated a new method of measuring the activity statement of TrA. We studied any relationship between the findings of the standard ultrasound scan and the new method of measuring the fluctuations of waist circumference. The thickness of TrA was measured by ultrasound, was compared with the waist circumference in 19 participants. The relationship between the two sets of findings was clinically significant. Any increase in waist circumference was correlated with more muscle contraction. Because of some limitations of standard methods of muscle thickness measuring such as inability of the ultrasound probes for covering the full length of the muscle, the new method based on changes of waist circumference would be feasible choice for routine daily use.

\section{Introduction}

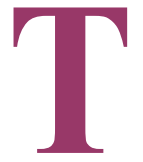

ransversus Abdominis (TrA) is a key muscle in core stability $[1,2]$. Different rehabilitation protocols target improving the timing and endurance of this muscle to recover spinal stability and ultimately prevent or alleviate back pain [3, 4]. Studies have shown that monitoring the activity of this muscle is not an easy task $[5,6]$ because of its deep location. Ultrasound is one method for monitoring the activity of $\operatorname{TrA}$. The reliability and validity of this method have been acceptable [7-10], but it is expensive, and its application depends on sufficient training. The pressure biofeedback unit is another device that has been used to achieve the same clinical objectives [11]. Few studies have examined the repeatability and validity of the device $[6,12]$. Furthermore, the problem with the cost and the required training persists.

$\operatorname{Tr} \mathrm{A}$ is a muscle with horizontal fibers. It creates an "internal corset" in conjunction with the abdominal and thoracolumbar fascia [13]. TrA is the only contractible element of this corset, and its contraction can decrease waist circumference. An established method for achieving isolated contraction of $\operatorname{TrA}$ is the so-called "hollowing maneuver" [14-16]. This technique involves drawing the abdominal wall in towards the spine without affecting breathing [16]; this is important as it can be challenging to contract TrA without simultaneous diaphragm contraction, and continued breathing shows that this is not occurring.
The purpose of this study was to investigate the relationship between the activity of $\operatorname{Tr} A$ and changes in waist circumference.

\section{Materials and Methods}

The study was approved by the Ethics Committee of the National Centre for Prosthetics and Orthotics (NCPO). A convenience sample of volunteers taken from the staff of Strathclyde University was recruited for this study. The required sample size was 18 based on $\alpha=0.05, \beta=$ 0.20 , for a pilot study involving five participants, where the minimum and maximum expected levels of reliability were 0.7 and 0.9 , respectively. The sample was increased to cover the potential dropout rate. Therefore, 19 participants were recruited. The exclusion criteria were neurological conditions affecting the abdominal muscles, sensory impairment in the trunk area, and change in an exercise routine during the previous 4 weeks before inclusion, low back pain episodes during the year before inclusion, pregnancy, previous abdominal or spinal surgery, inability to perform the hollowing maneuver following training, and previous training in the hollowing maneuver. All participants gave informed consent to participate in the study, following which they were given an appointment for training in the hollowing maneuver Training in the correct performance of the hollowing maneuver was carried out according to a standard protocol and approved by qualified physiotherapists. The participants were trained in four-point kneeling (quadruped), supine (hook-lying), chair seated, and upright standing as posture is essential in the recruitment of $\operatorname{Tr} A$ [17]. 
Table 1. Demographics of the study participants

\begin{tabular}{cccc}
\hline Variables & Min. & Max. & Mean \pm SD \\
\hline Weight $(\mathrm{kg})$ & 50.8 & 100.0 & $72.13 \pm 12.58$ \\
Height $(\mathrm{m})$ & 1.52 & 1.88 & $1.69 \pm 0.09$ \\
\hline BMI $\left(\mathrm{kg} / \mathrm{m}^{2}\right)$ & 19.12 & 33.41 & $25.29 \pm 4.13$ \\
Age $(\mathrm{y})$ & 24 & 63 & $40.74 \pm 13.65$ \\
\hline
\end{tabular}

Iranian Rehabilitation Journa

To confirm the correct performance of the hollowing maneuver, an ultrasound scan was performed to display the thickness of the transversus abdominis muscle while the waist circumference was measured simultaneously. A diagnostic ultrasound imaging system (Shimasonic SDU-400, Shimadzu Corporation, Kyoto, Japan) was used with a linear probe (nominal frequency: $5 \mathrm{MHz}$, view width: $61 \mathrm{~mm}$ ). The maximum thickness of TrA was measured from an ultrasound image. The images were taken from the anterolateral aspect of the abdominal wall with the subject in a standing position. The gel was interposed between the transducer and the skin, and the transducer was positioned adjacent and perpendicular to the abdominal wall in a horizontal orientation on the anterolateral aspect of the abdomen halfway between the iliac crest and inferior border of the rib cage [9]. The angle of the probe was manipulated until a clear image of the lateral abdominal muscles (TrA, internal oblique, and external oblique) was identified. Once the ideal picture was generated, a still image was captured. All measurements were taken from that still image. Before assessing the thickness of the TrA, the ability of the investigator to conduct the ultrasound evaluation was confirmed by a qualified sonographer. This procedure involved measuring the thickness of TrA on five participants on two separate occasions, once by the observer and once by the qualified sonographer, and the results were compared clinically. The sonographer then confirmed the ability of the observer to identify TrA accurately and to measure its thickness. To measure the waist circumference, a plastic tape measure was placed around the participant's bare abdomen, just one inch above the iliac crests. Care was taken to ensure that the tape was snug but did not compress the skin and was parallel to the floor while the participant was standing [18].

\section{Results}

Of 43 people who responded to the recruitment letter, 23 met the eligibility criteria; the first 15 (convenience sample) were selected for the study. One person decided to withdraw from the study and did not show at the appointment. The data from these 14 participants were combined with data from 5 participants in the pilot study, as the recruitment criteria were similar between the pilot and the main study. So we have a total of 19 participants in the final study (10 males, 9 females). The demographics of the participants are presented in Table 1. The descriptive statistics related to the test variables are presented in Table 2.

The distribution of the quantitative variables was verified using the Kolmogorov-Smirnov test, and normality was assumed for all the quantitative variables.

Table 2. Descriptive statistics of the test variables in the study

\begin{tabular}{cccc}
\hline Parameters & Min. & Max. & Mean \pm SD \\
\hline Waist Circumference (relaxed) [19] & 69.0 & 109.0 & $89.6 \pm 10.3$ \\
Waist Circumference (contracted) [19] & 65.0 & 102.0 & $84.4 \pm 9.8$ \\
Changes in waist circumference [19] & 2.0 & 8.0 & $5.2 \pm 1.7$ \\
TrA thickness (relaxed) [20] & 6 & 8 & $11.7 \pm 0.6$ \\
TrA thickness (contracted) [20] & 8 & 5 & $10.0 \pm 0.8$ \\
Changes in thickness of TrA [20] & 1 & $5.5 \pm 1.0$ \\
\hline
\end{tabular}


Table 3. The Pearson correlation between changes in the waist circumference and changes in the thickness of TrA

\begin{tabular}{cccc}
\hline \multicolumn{1}{c}{ Parameters } & & Waist Circumference & TrA Thickness \\
\hline \multirow{2}{*}{ Waist circumference } & Correlation & 1 & -0.710 \\
& Sig. & NS & 0.001 \\
TrA thickness & $\mathrm{n}$ & 19 & 19 \\
& Correlation & -0.710 & 1 \\
& Sig. & 0.001 & 19
\end{tabular}

To investigate a possible linear relationship between changes in the thickness of the TrA muscle measured by ultrasound and changes in the waist circumference, we used the Pearson correlation test. According to the null hypothesis, there is no linear relationship between changes in the thickness of the TrA and changes in the waist circumference during the performance of a successful hollowing maneuver. The result of the Pearson correlation (Table 3) was significant at the 0.01 level (2-tailed), with $\mathrm{r}$ as -0.710 . The result indicated a good, inverse relationship between these two variables.

\section{Discussion}

The transversus abdominis muscle wraps around the waist area. With attachments to the lumbosacral fascia and abdominal fascia, it creates an internal corset [13] when contracted. Concentric contraction in this muscle, as with any other skeletal muscle, results in a decrease in length. When skeletal muscle contracts, actin, and myosin filaments slide over each other to increase their overlaps and create more cross-bridges which shorten the muscle length; consequently, the contraction of transversus abdominis, which encircles the waist, will result in a decrease in the waist circumference. Richardson confirms this by explaining that "if the transversus abdominis contracts bilaterally, it reduces the circumference of the abdominal wall and flattens the abdominal wall" [13]. This finding is in line with the results of the current study, which shows that changes in waist circumference can be an indicator for activity of the transversus abdominis.

The next question would be how reliable and repeatable the waist circumference measurements are. The protocol of measuring waist circumference, which was employed in this study, has been suggested by Chen, who conducted a study on 4732 participants in Scotland [21]. Chen had already reported the intra-observer and inter- observer reliability of his method by performing a study on a larger scale, including 218121 participants who were given two measurements within a week of each other by two observers [18]. The intra-observer Intra-class Correlation Coefficient (ICC) for measuring waist circumference has been determined as 0.987 (95\% confidence interval [5]: 0.983 to 0.990$)$ and the inter-observer ICC for waist circumference 0.988 (95\% CI: 0.982 to 0.993 ) [18]. It can be asked whether contraction of any other muscle can produce the same reduction in waist circumference. The other muscles that can considerably contribute to the changes in the waist circumference are the diaphragm and intercostal muscles. If someone holds their breath and expands their ribcage, they can create negative pressure inside their abdominal cavity and, as a consequence, decrease the waist circumference. That is why the correct performance of the hollowing manoeuver requires that the person continues breathing normally.

Monitoring the activity of TrA based on changes of waist circumference would be more feasible for routine daily use than one based on changes in the thickness of the transversus abdominis, as assessed by ultrasound, or based on the changes in the weight of abdominal contents when one lies prone, as assessed by a pressure biofeedback unit.

About the weight, the same health survey for England gives $71.3 \mathrm{~kg}$ (for women), $85.7 \mathrm{~kg}$ (for men) and 78.50 $\mathrm{kg}$ (in total) for all people aged between 25 and 64 years. The corresponding figures for the study participants were $68.4,75.5$, and $72.13 \mathrm{~kg}$, respectively. This result means that the participants in this study were on average $6.37 \mathrm{~kg}$ lighter than the general population in England. This difference is statistically significant (1-sample $\mathrm{t}$ test, $\mathrm{P}=0.041$ ). Thus, the participants in the current study did not present the same characteristics as the general population, and therefore any quantitative generalization needs to be carried out with caution. 
Ultrasound was chosen as the gold standard for this purpose $[8,22,23]$. As stated earlier, the pressure biofeedback unit lacks the required reproducibility. Magnetic resonance imaging (MRI) was neither practical at the time nor was this technique within the study's budget. However, a recent study using a sample size of 13 healthy participants who have undergone MRI and ultrasound imaging while performing the hollowing maneuver demonstrated that the ICC coefficients for measures of muscle thickness obtained by MRI and ultrasound were high within the range of 0.78 to 0.95 [19]. This finding confirms that ultrasound has excellent validity for monitoring the activity of the TrA.

The ultrasound scan, which is the "gold standard" for monitoring the activity of this muscle, measures changes in TrA thickness when contracted. Therefore, as muscle activity is compared through measurement by two different instruments using two separate scales, the intra-class correlation was not a suitable statistical method, and the Pearson correlation was employed instead. This test is advocated as a statistical method for assessing a linear relationship between two activity measurements on separate scales. The correlation was shown to be significant at the 0.01 level (2-tailed), having $\mathrm{r}$ as -0.71. A negative $\mathrm{r}$ was expected beforehand as the contraction of the TrA decreases the waist circumference but increases the thickness of the muscle.

As stated previously, the pressure biofeedback unit senses any decrease in the contact pressure when the user is lying prone over the unit and performs the hollowing maneuver. The pressure over the unit comes from the weight of the trunk area and particularly abdominal contents. The weight of the thoracic area transfers to the supporting plate through the ribcage, and the weight of the pelvic region transfers to the supporting plate through the anterior superior iliac spines and possibly through the symphysis pubis. When the TrA muscle contracts, its connection to the lumbar vertebrae (origin) acts as a static end, and its connection to the abdominal fascia (insertion) acts as a dynamic end. So a concentric contraction will pull the abdominal wall and the abdominal contents up, which will decrease the pressure on the unit. This means that using the pressure biofeedback unit, the function of the muscle is measured indirectly, which may be another reason for the poor reproducibility of the unit [6].

The ultrasound scanner monitors the activity of the muscle based on the change in thickness. Concentric contraction of skeletal muscle results in a reduction in muscle length and increases the thickness of the muscle. Measurement of this change in thickness is the method advised for monitoring the activity of the TrA, as mea- suring the thickness of the muscle is more practicable because the ultrasound probes are not long enough to cover the length of the transversus abdominis. Additionally, the muscle follows a curve and cannot be fully monitored using a linear probe. Conversely, the thickness of the muscle is relatively easy to measure as the probe acts tangentially to the curve of the muscle and, therefore, can measure its actual thickness.

One of the limitations of this study was the small sample size with its heterogeneous participants that is insufficient for the generalization of the results. The authors recommend doing this study with larger sample size. Also, waist circumference might be affected by some factors such as digestion, which should be considered whether it is negligible or not.

\section{Conclusion}

The thickness of the TrA, produced by a correctly executed hollowing maneuver, is highly correlated with changes in the waist circumference.

\section{Ethical Considerations}

\section{Compliance with ethical guidelines}

All ethical principles are considered in this article. The participants were informed of the purpose of the research and its implementation stages. They were also assured about the confidentiality of their information and were free to leave the study whenever they wished, and if desired, the research results would be available to them. A written consent has been obtained from the subjects. principles of the Helsinki Convention was also observed.

\section{Funding}

This research did not receive any grant from funding agencies in the public, commercial, or non-profit sectors.

\section{Authors' contributions}

All authors equally contributed to preparing this article.

\section{Conflict of interest}

The authors declared no conflict of interest.

\section{Acknowledgments}

We would like to acknowledge the contribution of the following persons: Angus McFadyen, Reader in Health Statistics at Glasgow Caledonian University, for offering 
supportive comments through the statistical analyses; Janet Bonn, Kay Keir, and Tom McCarron, from Division of Physiotherapy, Glasgow Caledonian University, for their vital physiotherapy input to this project; Claire Bowman, from Southern General Hospital, for her valued contribution in working with ultrasound.

\section{References}

[1] Allison GT, Morris SL. Transversus abdominis and core stability: Has the pendulum swung? British Journal of Sports Medicine, 2008; 42(11):630-1. [DOI:10.1136/bjsm.2008.048637] [PMID]

[2] Hodges PW. Core stability exercise in chronic low back pain. Orthopedic Clinics of North America. 2003; 34(2):24554. [DOI:10.1016/S0030-5898(03)00003-8]

[3] Jammett R. Spinal stabilization: The new science of back pain. Halifax: RMJ Fitness \& Rehabilitation Consultants; 2001 https://books.google.com/books?id=pV0yAAAACAAJ\&dq

[4] Barr KP, Griggs M, Cadby T. Lumbar stabilization: Core concepts and current literature, Part 1. American Journal of Physical Medicine \& Rehabilitation. 2005; 84(6):473-80. [DOI:10.1097/01.phm.0000163709.70471.42] [PMID]

[5] Critchley DJ, Coutts FJ. Abdominal muscle function in chronic low back pain patients: Measurement with realtime ultrasound scanning. Physiotherapy. 2002; 88(6):322-32. [DOI:10.1016/S0031-9406(05)60745-6]

[6] Storheim, K, Bø K, Pederstad O, Jahnsen R. Intra-tester reproducibility of pressure biofeedback in measurement of transversus abdominis function. Physiotherapy Research International, 2002; 7(4):239-49. [DOI:10.1002/pri.263] [PMID]

[7] McMeeken JM, Beith ID, Newham DJ, Milligan P, Critchley DJ. The relationship between EMG and change in thickness of transversus abdominis. Clinical Biomechanics. 2004 19(4):337-42. [DOI:10.1016/j.clinbiomech.2004.01.007] [PMID]

[8] Norasteh A, Ebrahimi E, Salavati M, Rafiei J, Abbasnehad E. Reliability of B-mode ultrasonography for abdominal muscles in asymptomatic and patients with acute low back pain. Journal of Bodywork and Movement Therapies. 2007 11(1):17-20. [DOI:10.1016/j.jbmt.2005.11.002]

[9] Bunce SM, Hough AD, Moore AP. Measurement of abdominal muscle thickness using M-mode ultrasound imaging during functional activities. Manual Therapy. 2004; 9(1):41-4. [DOI:10.1016/S1356-689X(03)00069-9]

[10] Koppenhaver SL, Hebert JJ, Fritz JM, Parent EC, Teyhen DS, Magel JS. Reliability of rehabilitative ultrasound imaging of the transversus abdominis and lumbar multifidus muscles. Archives of Physical Medicine and Rehabilitation. 2009; 90(1):87-94. [DOI:10.1016/j.apmr.2008.06.022] [PMID]

[11] Cairns MC Harrison K, Wright C. Pressure biofeedback: A useful tool in the quantification of abdominal muscular dysfunction? Physiotherapy. 2000; 86(3):127-38. [DOI:10.1016/ S0031-9406(05)61155-8]
[12] von Garnier K, Köveker K, Rackwitz B, Kober U, Wilke $\mathrm{S}$, Ewert T, et al. Reliability of a test measuring transversus abdominis muscle recruitment with a pressure biofeedback unit. Physiotherapy. 2009; 95(1):8-14. [DOI:10.1016/j.physio.2008.10.003] [PMID]

[13] Richardson C, Hodges PW, Hides J. Therapeutic exercise for lumbopelvic stabilization. London: Churchill Livingstone; 2004. https://books.google.com/ books?id=32JsAAAAMAAJ\&q

[14] Bjerkefors A, Ekblom MM, Josefsson KK, Thorstensson A Deep and superficial abdominal muscle activation during trunk stabilization exercises with and without instruction to hollow. Manual Therapy. 2010; 15(5):502-7. [DOI:10.1016/j. math.2010.05.006] [PMID]

[15] Urquhart DM, Hodges PW, Story IH. Postural activity of the abdominal muscles varies between regions of these muscles and between body positions. Gait \& Posture. 2005; 22(4):295-301. [DOI:10.1016/j.gaitpost.2004.09.012] [PMID]

[16] Barnett F, Gilleard W. The use of lumbar spinal stabilization techniques during the performance of abdominal strengthening exercise variations. The Journal of Sports Medicine and Physical Fitness. 2005; 45(1):38-43. [PMID]

[17] Reeve A, Dilley A. Effects of posture on the thickness of transversus abdominis in pain-free subjects. Manual Therapy. 2009; 14(6):679-84. [DOI:10.1016/j.math.2009.02.008] [PMID]

[18] Chen MM, Lear SA, Gao M, Frohlich JJ, Birmingham CL Intraobserver and interobserver reliability of waist circumference and the waist-to-hip ratio. Obesity Research. 2001; 9(10):651. [DOI:10.1038/oby.2001.87] [PMID]

[19] Hides J, Wilson S, Stanton W, McMahon S, Keto H, McMahon K, et al. An MRI investigation into the function of the transversus abdominis muscle during "drawing-in" of the abdominal wall. Spine. 2006; 31(6):E175-8. [DOI:10.1097/01. brs.0000202740.86338.df] [PMID]

[20] Ecker ML, Betz RR, Trent PS, Mahboubi S, Mesgarzadeh $\mathrm{M}$, Bonakdapour A, et al. Computer tomography evaluation of Cotrel-Dubousset instrumentation in idiopathic scoliosis. Spine. 1988; 13(10):1141-4. [DOI:10.1097/00007632-19881000000015] [PMID]

[21] Chen R, Tunstall-Pedoe H. Socioeconomic deprivation and waist circumference in men and women: The Scottish MONICA surveys 1989 -1995. European Journal of Epidemiology. 2005; 20(2):141-7. [DOI:10.1007/s10654-004-4498-y] [PMID]

[22] Whittaker JL. Ultrasound imaging for rehabilitation of the lumbopelvic region: A clinical approach. London: Churchill Livingstone; 2007. [DOI:10.1016/B978-0-443-06856-0.X5001-5]

[23] Vasseljen O, Fladmark AM, Westad C, Torp HG. Onset in abdominal muscles recorded simultaneously by ultrasound imaging and intramuscular electromyography. Journal of Electromyography and Kinesiology. 2009; 19(2):e23-31. [DOI:10.1016/j.jelekin.2007.07.013] [PMID] 